\title{
Fingertip force control during bimanual object lifting in hemiplegic cerebral palsy
}

\author{
Bert Steenbergen · Jeanne Charles $\cdot$ Andrew M. Gordon
}

Received: 21 May 2007 / Accepted: 13 November 2007 / Published online: 26 January 2008

(C) The Author(s) 2008

\begin{abstract}
In the present study we examined unimanual and bimanual fingertip force control during grasping in children with hemiplegic cerebral palsy (CP). Participants lifted, transported and released an object with one hand or both hands together in order to examine the effect on fingertip force control for each hand separately and to determine whether any benefit exists for the affected hand when it performed the task concurrently with the less-affected hand. Seven children with hemiplegic CP performed the task while their movement and fingertip force control were measured. In the bimanual conditions, the weight of the instrumented objects was equal or unequal. The durations of the all temporal phases for the less-affected hand were prolonged during bimanual control compared to unimanual control. We observed close synchrony of both hands when the task was performed with both hands, despite large differences in duration between both hands when they performed separately. There was a marginal benefit for two of the five force related variables for the affected hand (grip force at onset of load force, and peak grip force) when it transported the object simultaneously with the less-affected hand. Collectively, these results corroborate earlier findings of reaching studies that showed slowing down of the
\end{abstract}

B. Steenbergen $(\square)$

NICI (Nijmegen Institute for Cognition and Information),

Radboud University Nijmegen, PO Box 9104,

6500 HE Nijmegen, The Netherlands

e-mail: steenbergen@ nici.ru.nl

J. Charles · A. M. Gordon

Department of Biobehavioral Sciences,

Teachers College, Columbia University,

New York, USA less-affected hand when it moved together with the affected hand. A new finding that extends these studies is that bimanual tasks may have the potential to facilitate force control of the affected hand. The implications of these findings for recent rehabilitative therapies in children with $\mathrm{CP}$ that make use of bimanual training are discussed.

\section{Introduction}

Impaired hand function is a major debilitating factor for the performance of activities of daily living in hemiplegic cerebral palsy $(\mathrm{CP})$. There are a number of studies demonstrating impairments of the affected upper extremity compared to the less-affected upper extremity, including general slowness of movement (e.g., Brown et al. 1989; Utley and Sugden 1998), discontinuous movements (e.g., Chang et al. 2005), variable hand trajectories (e.g., van Thiel et al. 2002), increased levels of trunk involvement (e.g., van Roon et al. 2004), inappropriate coordination of grasping forces (e.g., Eliasson et al. 1991; Forssberg et al. 1999; Gordon et al. 1999), and deficits in anticipatory motor planning (e.g., Eliasson et al. 1992; Gordon and Duff 1999; Duff and Gordon 2003; Mutsaarts et al. 2006; see Steenbergen and Gordon 2006 for review). Potentially, the affected hand may benefit when it performs movements in co-operation with the less-affected hand. That is, the movement asymmetry between the performance of the two hands that is present when moving unimanually may decrease when moving bimanually through a specific facilitation of the affected hand. Still, studies on bimanual reaching and grasping movements in young adolescents with hemiplegic $\mathrm{CP}$ are equivocal with regard to this alleged facilitation of the affected side under simultaneous movement conditions. While some benefit has been shown when individuals with 
CP make upper limb movements at a self-selected pace (e.g. Sugden and Utley 1995; Volman et al. 2002), benefits for the affected side were absent when movements were made under fast-as-possible conditions (Utley and Sugden 1998; Steenbergen et al. 1996; Steenbergen et al. 2000; see Gordon and Steenbergen, in press for review). van Thiel and Steenbergen (2001) have argued that the extent to which bimanual movement facilitates control of the affected extremity may be dependent upon the movement characteristics. Similarly, bimanual coupling and facilitation may also be dependent on the type of task. During fast hitting tasks with a hand-held rod that involves mainly proximal movements coupling was stronger compared to grasping that also involved isometric force increase of the fingers (van Thiel and Steenbergen 2001). Based on this result and the assumption that distal musculature are thought to receive minimal input from bilateral pathways (Brinkman and Kuypers 1973; Colebatch and Gandevia 1989), it may be hypothesized that facilitation of the affected hand will be absent when bimanual tasks are performed that require fingertip force control. At odds with this hypothesis, however, are the results of Utley and Sugden (1998: for a review see Utley and Steenbergen 2006). They compared unimanual and bimanual control in children with hemiplegic CP performing three tasks with increasing distal involvement (from reaching to grasping). Movement duration of the affected side decreased when moving bimanually as compared to when moving unimanually. The extent to which distal control is affected when moving bimanually has yet to be directly tested.

There is ample evidence of disturbances in planning and timing of fingertip force control in the affected hand in hemiplegic CP (e.g., Eliasson et al. 1991, 1992; Gordon et al. 1999; Kutz-Buschbeck et al. 2000). Importantly, however, several studies have shown that initial lifts of an object with the less-affected hand can facilitate subsequent performance with the affected hand (Gordon et al. 1999, 2006a). It is not known whether deficits of fingertip force control in the affected hand are ameliorated when tasks are performed simultaneously with both hands. Such knowledge is essential as it provides important information on how to structure practice during bimanual training (Charles and Gordon 2006; Gordon et al. 2007), and is the focus of the present study. Based on the potential of the less-affected hand to facilitate subsequent force control of the affected hand, we hypothesize fingertip force control to be facilitated in the affected extremity during simultaneous performance. In addition, with respect to timing of the action, the less-affected hand may simply slow down to match the grasp speed of the affected hand, as was shown before during reaching. In order to test these hypotheses we analysed the durations of the different phases of fingertip force control as well as relevant force related parameters.

\section{Method}

\section{Participants}

Seven male children with hemiplegic CP (age 4.513.6 years) participated in the present study (see Table 1 for participant information). The children were recruited from area schools and clinics. Children were chosen who had the ability to grasp and lift a small object between the fingertips, were mainstreamed in the schools and generally had normal cognitive abilities according to the Kaufman Brief Intelligence Test (Kaufman and Kaufman 1990), and could follow instructions. In addition, dexterity of each child was assessed using the Jebsen-Taylor Test of Hand Function (Jebsen et al. 1969), which measures the time to complete a battery of unimanual activities. Note that the times for the non-involved hand are longer than that seen during typical development (Jebsen et al. 1969). The Assisting Hand Assessment (AHA: Krumlinde-Sundholm and Eliasson 2003; Krumlinde-Sundholm et al. 2007) was used to measure and describe the effectiveness with which a child with a unilateral disability makes use of his/her affected hand (assisting hand) in performance of bimanual activities. Six of the children had right hemiparesis while the seventh child had left hemiparesis. Sensory discriminative capacity of the index finger and thumb was assessed via administration of the two point discrimination task (Mackinnon and Dellon 1985). None of the children exhibited overt mirror movements during unimanual movements. Informed consent was obtained from all participating children and their parents and the study was approved by the Teachers College, Columbia University Institutional Review Board.

\section{Apparatus}

Two visually identical grip instruments were employed which allowed measurement of grip (normal) and load

Table 1 Participant information

\begin{tabular}{|c|c|c|c|c|c|c|}
\hline Child & $\begin{array}{l}\text { Involved } \\
\text { side }\end{array}$ & Gender & $\begin{array}{l}\text { Age } \\
\text { (years) }\end{array}$ & $\begin{array}{l}\text { Jebsen } \\
(\mathrm{A} / \mathrm{LA}, \mathrm{s})\end{array}$ & AHA & $\begin{array}{l}\text { TPD } \\
(\mathrm{A} / \mathrm{LA}, \mathrm{mm})\end{array}$ \\
\hline DS & Right & Male & 13.5 & $505 / 68$ & 59 & $2 / 4$ \\
\hline LM & Right & Male & 7.3 & $129 / 85$ & 58 & $5 / 3$ \\
\hline EM & Right & Male & 13.6 & $361 / 43$ & 70 & $5 / 3$ \\
\hline KD & Right & Male & 8.8 & $507 / 55$ & 55 & $13 / 3$ \\
\hline JM & Right & Male & 7.8 & $196 / 40$ & 65 & $4 / 3$ \\
\hline KM & Right & Male & 4.5 & $77 / 53$ & 81 & $2 / 2$ \\
\hline JA & Left & Male & 7.3 & $161 / 63$ & 66 & $7 / 3$ \\
\hline
\end{tabular}

Note Age in years and months, Jebsen Jebsen-Taylor Test of Hand Function (lower times mean better performance), AHA Assisting Hand Assessment (out of a possible score of 88 , higher scores mean better performance), TPD Two Point Discrimination, A Affected side, $L A$ Less Affected side 
(tangential) forces from the thumb and index finger of each hand. The forces at each contact were measured using force transducers (Mini and Nano $F / T$ transducers, ATI Industrial Automation, NC; $F x$ and $F y, 0.025 \mathrm{~N}$ resolution). The sensors were covered with a smooth plastic surface $(4.5 \mathrm{~cm}$ diameter, $4.4 \mathrm{~cm}$ apart) to make the size of the grasp surface identical regardless of the size of the transducer. The position of each object was measured using electromagnetic position-angle sensors (Pohlemus Fastrack, Colchester, VT; $0.075 \mathrm{~mm}$ resolution). The weight of the objects could be altered by inserting different masses in the base to adjust the weight to either $250 \mathrm{~g}$ or $500 \mathrm{~g}$ without altering their visual appearance. The force and position signals were sampled at 400 and $60 \mathrm{~Hz}$, respectively, using a flexible data acquisition and analysis system (SC/ZOOM, Umeå University, Sweden).

\section{Procedure}

Prior to the experiment, all children washed their hands to remove sweat and excessive oil from the skin. They sat on a chair in front of a table, adjusted in height to position the forearm approximately horizontal to and above the table when the object was grasped. The grip instruments were aligned with each shoulder. Children were instructed to grasp the object(s), either unimanually or bimanually (i.e., simultaneously), between the thumb and the index finger ("precision grip") of each hand, lift and transport them and release them onto a shelf $(8 \mathrm{~cm}$ high) located $15 \mathrm{~cm}$ anterior to the start position. This task was chosen since it is discrete with a clear onset and completion point, easy to understand and does not require instruction in regards to how to perform the task. After releasing the object on the shelf the experimenter then returned the instruments to their start position prior to the next trial. The task was performed at self pace. In the bimanual conditions, the child was simply told to perform the task with both hands, without explicit emphasis on symmetry of performance. We had four reasons to chose this particular instruction and task; (1) transport provides a clear, goal-oriented instruction whereby the task dictates what to do and that is related to tasks in daily life, (2) if these young participants would have been instructed to lift and hold both objects $5 \mathrm{~cm}$, this would have required too much attention and our expectation was that this young group would invariably not do that accurately, (3) we wanted to use a task that resembled previous studies on this topic as closely as possible to allow for relevant comparisons among studies (e.g., Steenbergen et al. 2000; Utley and Sugden 1998), and (4) lift and hold has partially been studied by Kuhtz-Buschbeck et al. (2000).

Participants performed a series of 40 lifts of the instrumented objects. After every five lifts the objects were placed behind a screen and the experimenter removed the masses and either replaced them with the same or the alternative weight. The lifting series resulted in five consecutive lifts for each of eight conditions (order randomized across participants) that were determined via manipulation of the Hand used (affected or less-affected), object Weight (250 g or $500 \mathrm{~g}$ ), and movement Condition (unimanual or bimanual). Specifically, each participant performed five trials in the following unique conditions, (1) unimanual affected $250 \mathrm{~g}$, (2) unimanual affected $500 \mathrm{~g}$, (3) unimanual lessaffected $250 \mathrm{~g}$, (4) unimanual less-affected $500 \mathrm{~g}$, (5) bimanual affected 250 g_less-affected $250 \mathrm{~g}$, (6) bimanual affected 500 g_less-affected $500 \mathrm{~g}$, (7) bimanual affected 250 g_less-affected $500 \mathrm{~g}$, (8) bimanual affected 500 g_lessaffected $250 \mathrm{~g}$.

\section{Data acquisition and analysis}

The signals from the instrument were digitized with 12 bits resolution, and stored in a flexible laboratory computer system (SC/ZOOM, Department of Physiology, Umeå University, Sweden). Figure 1 shows typical force and position traces from the less-affected hand of a child with CP while performing the task, along with the measures examined. There is often a short delay between finger and thumb contact (T0-T1), defined as grip force $>0.1 \mathrm{~N}$ (finger difference). During the preload phase (T1-T2), there is a small grip force increase until the onset of positive load force increase ( $\mathrm{T} 2$, defined as load force $>0.1 \mathrm{~N}$ ), after which the isometric grip and load force increase in parallel during the loading phase (T2-T3). The end of this phase (T3) is signified when the load force overcomes the gravitational force

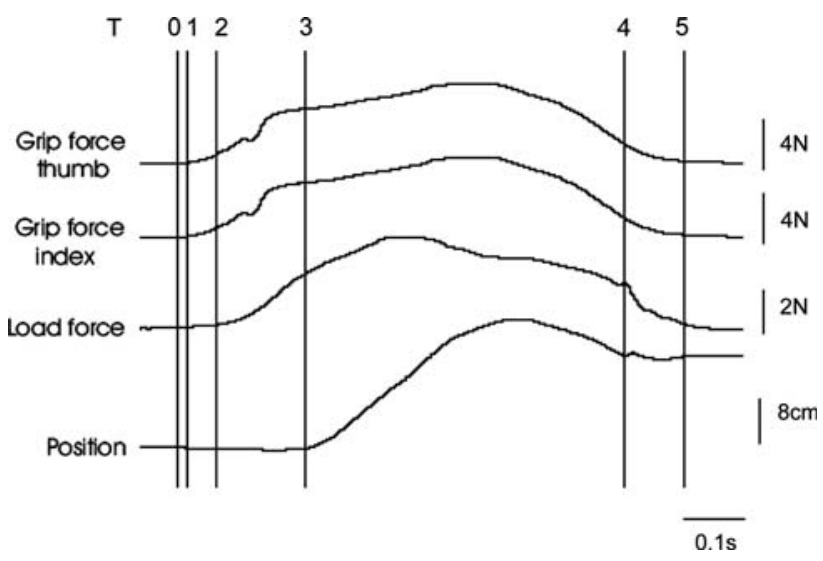

Fig. 1 Grip force, load force, and position traces from the less-affected hand of a child with CP while performing the task, along with the measures examined: finger difference (T0-T1) preload phase (T1-T2), onset of positive load force increase (T2), loading phase (T2-T3), transport phase T3-T4), replacement phase (T4-5). Total movement time is defined as the duration between $\mathrm{T} 0$ and $\mathrm{T} 5$ and signifies the duration of the complete grasp, transport and release movement. For elaborate description of the phases, see text 
on the object and the object is lifted from its support (defined as position $>1 \mathrm{~mm}$ ). Force increase then terminates. Subsequently, the object is transported to the desired position (transport: T3-T4), replaced (T4) and released (T5) onto the shelf. The duration between placement of the object down (T4) and release of the object (T5) signifies the release phase. Total movement time is defined as the duration between $\mathrm{T} 0$ and $\mathrm{T} 5$ and signifies the duration of the complete grasp, transport and release movement.

We performed analyses on two aspects of the data, one related to the temporal characteristics, and one related to the force characteristics of the movement. With respect to the temporal analysis we recorded the time at seven discrete events relative to object contact. The times were recorded at the points of: (1) load-force onset, (2) peak grip-force rate, (3) peak load-force rate, (4) object lift-off, (5) peak gripforce, (6) replacement, and (7) release. With respect to the force-related analysis, we extracted five force-related variables. The first variable, peak grip force rate $(\mathrm{d} G F / \mathrm{d} t)$ was calculated using a \pm 10 point numerical differentiation (i.e., calculated within a $\pm 25 \mathrm{~ms}$ window) and signifies the anticipatory planning of fingertip force. The second variable, grip force at load force onset (at T2, Fig. 1), is a measure of the sequencing of the two forces. The final three force-related variables were measured during the transport phase. These are peak grip force, average grip force, and variability of grip force (coefficient of variation; mean divided by the standard deviation taken from each sampled point $\times 100$ ). An interactive graphics terminal was used to indicate the temporal and force events described above.

The averages of each of these variables were used for data analyses. We performed two separate analyses. First, to determine whether the temporal and force parameters were different between unimanual and bimanual (uniform weight) conditions, we performed a 2 Condition (unimanual, bimanual) $\times 2$ Hand (affected, less-affected) $\times 2$ Weight $(250 \mathrm{~g}, 500 \mathrm{~g})$ repeated measures ANOVA on conditions 1-6 (i.e., with uniform weight). Second, to determine whether object weight in one hand influenced the temporal and force parameters in the other hand under bimanual conditions, we compared the uniform weight and non-uniform weight bimanual conditions (conditions 5-8) using a 2 Condition (bimanual equal-weight, bimanual unequal-weight) $\times 2$ hand (affected, less-affected) $\times 2$ weight $(250 \mathrm{~g}, 500 \mathrm{~g})$ repeated measures ANOVA. For analysis of the temporal aspects, Phase was included as a between-factor in the analysis (seven levels). For a similar two-step analysis procedure, see Kelso et al. (1979) and Steenbergen et al. (1996). Post-hoc tests were performed using Newman-Keuls procedures. Significance was considered at the $P<0.05$ level.

\section{Results}

Figure 2 shows representative grip force, load force and position traces from each hand under unimanual and
Fig. 2 Examples of force profiles for the affected hand (left panels) and less-affected hand (right panels) in the unimanual conditions (upper panels) and bimanual equal-weight conditions (lower panels) when lifting objects of $250 \mathrm{~g}$. T0-T3 represents the isometric force increase, encompassing finger difference, preload, and load phases. T3-T4 represents transport phase, and T4-T5 represents the replacement phase. The total movement time is denoted T0-T5
More Affected
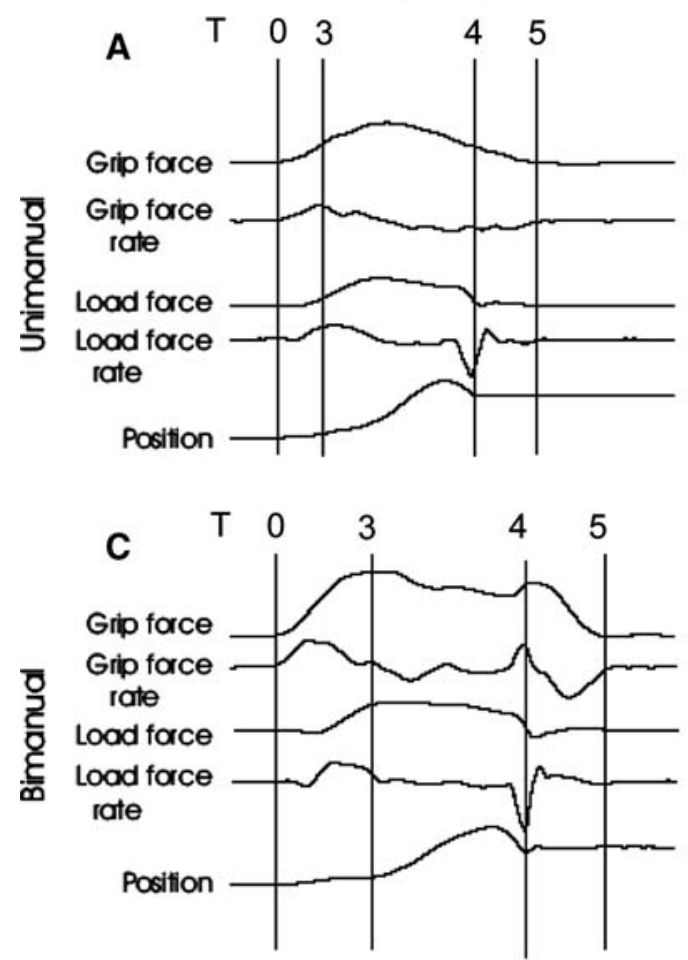

Less Affected
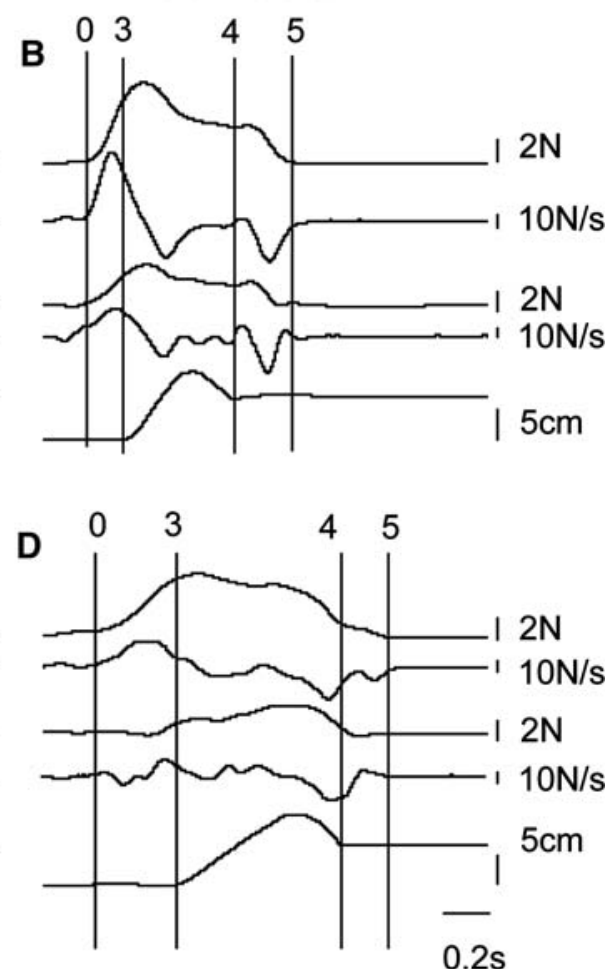
bimanual conditions for a child with CP (No. 5). As seen in the figure, the various phases are shorter in the less-affected hand during the unimanual conditions (A, B) as expected. Furthermore, when the two hands are compared, the durations of these phases are more similar during the bimanual conditions (C, D). However, what is striking from this figure is that the greater similarity between the two hands in the bimanual conditions was largely achieved by an increase in the duration of the preload/load phase (T0-3), transport (T3-4) and release (T4-5) in the bimanual conditions compared to the unimanual conditions. This is particularly evident when comparing the total movement times (T0-5). As shown in the example, for both hands total movement times were longer in the bimanual conditions compared to unimanual conditions; i.e., both hands slowed down under bimanual conditions. Note also that the peak (and subsequent) grip force during transport is higher in the less-affected hand. Below the statistical results are presented for the temporal variables first, followed by the force-related variables.

\section{Temporal aspects of the task}

Displayed in Fig. 3a-c are the durations of the seven phases of the movement in the unimanual conditions (a), bimanual equal-weight conditions (b), and bimanual unequal-weight conditions (c), for the affected and less-affected hand. It is clear that the time of the affected hand at each temporal event is prolonged compared to the less-affected hand during unimanual conditions (Fig. 3a). However, this timing difference between the two hands decreased to a large extent during bimanual conditions (Fig. 3b,c).

The first analysis showed main effects of condition $(F[1,42]=33.26, P<0.001)$ and weight $(F[1,42]=15.61$, $P<0.001)$, and a trend for a hand main effect $(F[1,42]=3.96, P=0.053)$. Post-hoc analysis of the significant condition $\times$ hand interaction $(F[1,42]=39.79$, $P<0.001)$ revealed that responses of the affected hand were slower than the less-affected hand only in the unimanual movement conditions. In the bimanual conditions movement durations of both hands were nearly identical, which was primarily due to the slowing down of the lessaffected hand (see Fig. 3b,c). Figure 4 displays total movement time of the complete task to illustrate the cumulative result of the slowing down of the less-affected hand during the bimanual movement conditions as compared to unimanual conditions. The lack of an influence of bimanual performance on the affected hand compared to unimanual conditions can also be seen in the figure.

The first analysis further revealed a hand $\times$ weight interaction $(F[1,42]=14.87, P<0.001)$, and a condition $\times$ phase interaction $(F[1,42]=2.94, P<0.05)$. Post-hoc analysis of the first interaction revealed that durations of both hands
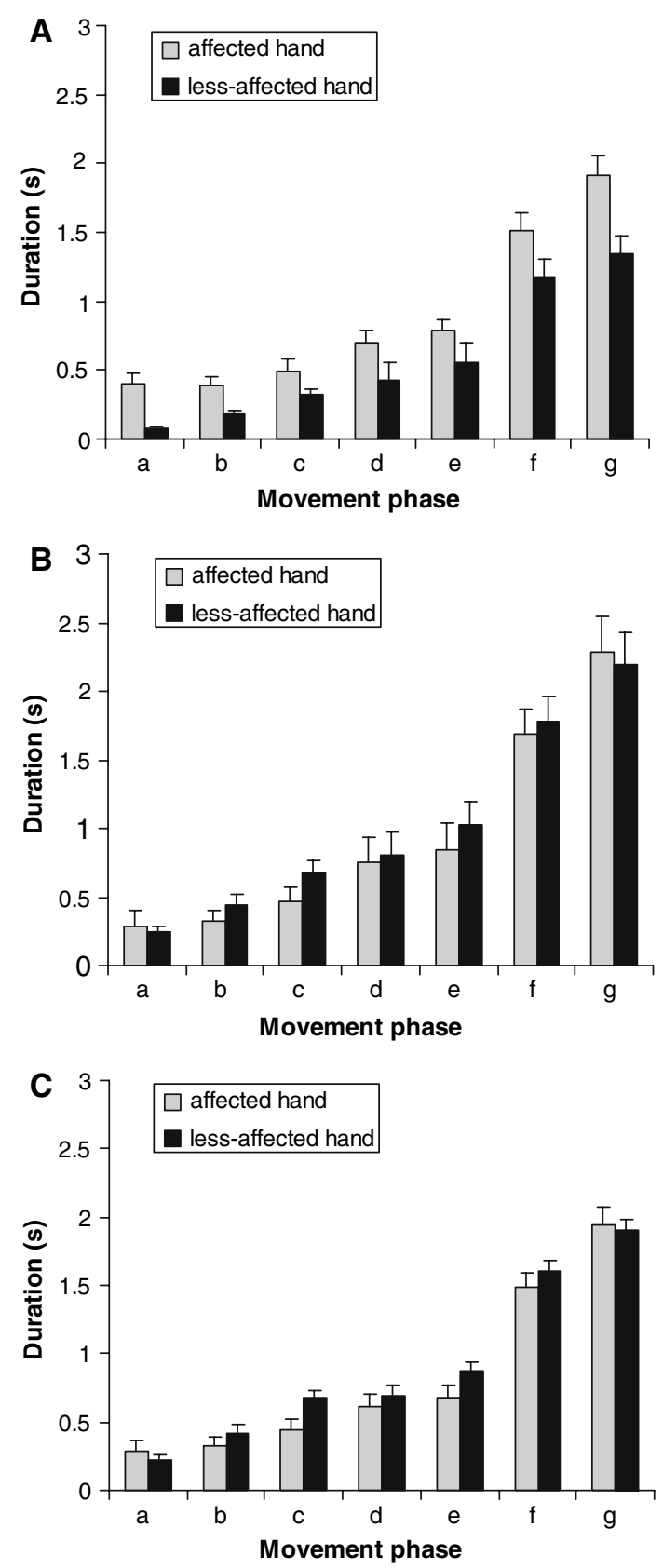

Fig. 3 Duration of the different movement phases for the affected and less-affected hand. Shown are the durations in the unimanual conditions (a), bimanual equal-weight conditions (b), and bimanual unequal-weight conditions (c). All durations are calculated relative to object contact (zero). These include load-force onset (a), peak gripforce rate (b), peak load-force rate (c), object lift-off (d), peak gripforce (e), replacement (f), and release (g). Error bars represent standard error

were similar for the light weight (affected $0.79 \mathrm{~s}$ versus less-affected $0.78 \mathrm{~s}$ ), but for the heavy weight durations were longer for the affected hand compared to the lessaffected hand $(1.05 \mathrm{~s}$ versus $0.83 \mathrm{~s})$. Post hoc analysis of the second interaction revealed that, except for the first time point (time of load force onset), the durations of all phases 


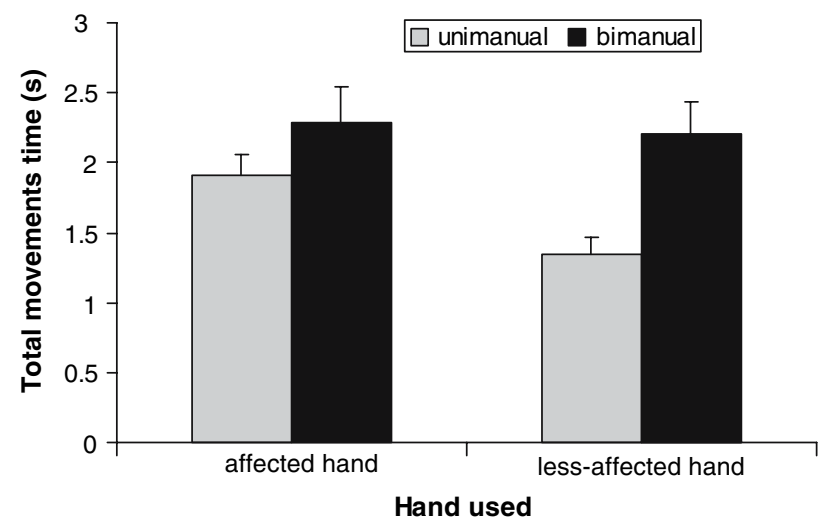

Fig. 4 Graphical display of the hand $\times$ condition interaction that was found for Total Movement Time. Error bars represent standard error

were longer in the bimanual conditions compared to unimanual conditions.

The second analysis only showed a main effect of weight $(F[1,42]=6.31, P<0.05$, where all durations were longer for the heavy weight compared to the light weight $(0.99 \mathrm{~s}$ versus $0.87 \mathrm{~s}$ ). There was a tendency for this weight effect on timing to disappear during the unequal weight conditions (but condition $\times$ weight interaction, $P>0.059$ ).

\section{Force-related aspects of the task}

Table 2 provides an overview of the five force-related parameters measured during the various conditions. To determine the sequencing of forces we measured the grip force at onset of load force. There was a higher grip force at load force onset for lifts with the heavier weight compared to lifts with the lighter weight, presumably reflecting anticipation $(F[1,6]=12.74, P<0.05 ; 6 / 7$ participants, exception participant $\mathrm{KM}$ ). Interestingly, there was a large difference in grip force at load force onset between both hands during unimanual conditions $(3.8 \mathrm{~N}$ affected hand versus $2.0 \mathrm{~N}$ less-affected hand), but this difference disappeared during bimanual conditions $(3.1 \mathrm{~N}$ affected hand versus $3.3 \mathrm{~N}$ less-affected, see Fig. 5; $F[1,6]=9.79$, $P<0.05 ; 6 / 7$ participants, exception participant KM). Thus, the grip force difference between the two hands during the bimanual conditions became more similar primarily by increasing the grip force at load force onset of the lessaffected hand in the bimanual conditions and slightly reducing grip force at load force onset of the affected hand during bimanual conditions. There was also a negative load force (pushing the object against the table surface) prior to load force onset, although the magnitude $(\sim 1 \mathrm{~N})$ was similar in all conditions).

Peak grip force rate (which occurred at the end of the preload phase) was higher for the heavier weight compared to the lighter one $(47.9 \mathrm{~N} / \mathrm{s}$ versus $39.5 \mathrm{~N} / \mathrm{s} ; F[1,6]=10.77$, $P<0.05 ; 6 / 7$ participants, exception participant $\mathrm{KM})$. In addition, it was different between both hands in unimanual conditions $(31.5 \mathrm{~N} / \mathrm{s}$ for the affected hand and $59.9 \mathrm{~N} / \mathrm{s}$ for the less-affected hand) but there was not a significant between-hand difference for this variable during the bimanual
Table 2 Values of the forcerelated parameters in the experimental conditions

\begin{tabular}{lccccc}
\hline Dependent variable & Affected hand & & & \multicolumn{2}{l}{ Less-affected hand } \\
\cline { 2 - 3 } \cline { 5 - 6 } & 250 $\mathrm{g}$ & $500 \mathrm{~g}$ & & $250 \mathrm{~g}$ & $500 \mathrm{~g}$ \\
\hline Unimanual movement conditions & & & & \\
Grip force at load force onset (N) & $3.14(0.69)$ & $4.37(0.98)$ & & $1.58(0.32)$ & $2.31(0.58)$ \\
Peak grip force rate (N/s) & $29.97(4.87)$ & $33.00(4.60)$ & & $53.18(8.48)$ & $66.64(9.27)$ \\
Peak grip force during transport (N) & $7.21(0.62)$ & $8.54(0.67)$ & & $9.57(1.38)$ & $13.49(1.25)$ \\
Mean grip force during transport (N) & $5.14(.33)$ & $6.56(.36)$ & & $6.91(.59)$ & $9.85(.51)$ \\
Variation in grip force during transport (\%) & $27.93(3.01)$ & $21.18(1.89)$ & & $23.14(2.38)$ & $21.04(2.21)$ \\
Bimanual equal-weight movement conditions & & & & & \\
Grip force at load force onset (N) & $2.69(0.83)$ & $3.45(0.85)$ & & $2.54(0.39)$ & $4.04(1.60)$ \\
Peak grip force rate (N/s) & $32.01(5.00)$ & $39.69(6.84)$ & & $42.92(7.33)$ & $52.21(6.58)$ \\
Peak grip force during transport (N) & $7.20(0.55)$ & $9.56(0.70)$ & & $10.09(1.47)$ & $15.20(2.23)$ \\
Mean grip force during transport (N) & $4.92(0.31)$ & $6.76(0.35)$ & & $7.49(0.73)$ & $12.60(1.35)$ \\
Variation in grip force during transport (\%) & $27.93(2.64)$ & $22.48(1.04)$ & & $25.05(3.82)$ & $16.75(0.98)$ \\
Bimanual unequal-weight movement conditions & & & & & \\
Grip force at load force onset (N) & $3.78(0.96)$ & $3.71(1.04)$ & & $2.70(1.13)$ & $2.25(0.59)$ \\
Peak grip force rate (N/s) & $34.71(5.41)$ & $37.51(4.06)$ & & $47.54(9.49)$ & $48.21(8.03)$ \\
Peak grip force during transport (N) & $7.96(0.73)$ & $9.52(0.53)$ & & $14.17(3.56)$ & $12.55(1.27)$ \\
Mean grip force during transport (N) & $5.48(0.31)$ & $6.84(0.27)$ & & $11.20(2.16)$ & $9.31(0.48)$ \\
Variation in grip force during transport (\%) & $26.80(1.85)$ & $25.84(1.66)$ & & $18.20(1.34)$ & $19.66(1.69)$ \\
\hline
\end{tabular}




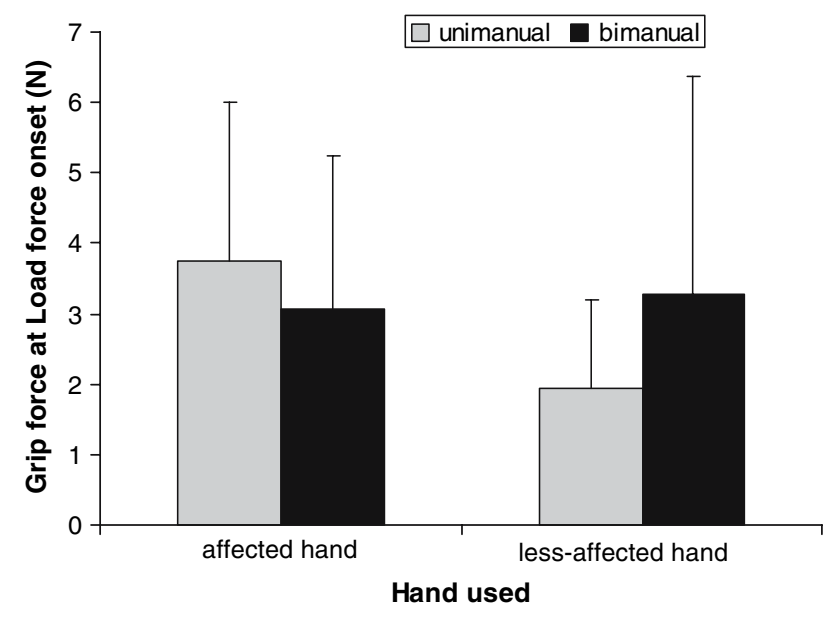

Fig. 5 Graphical display of the hand $\times$ condition interaction that was found for grip force at load force onset. Error bars represent standard error

conditions, as evidenced by post-hoc analysis of a significant hand $\times$ condition interaction $(F[1,6]=14.10$, $P<0.01 ; 35.9 \mathrm{~N} / \mathrm{s}$ for the affected hand and $47.6 \mathrm{~N} / \mathrm{s}$ for the less-affected hand, see Fig. 6; 6/7 participants, exception participant KM). Again, the difference between both hands decreased during bimanual conditions as compared to unimanual conditions, but not the same extent as compared to total movement time and grip force at load force onset.

The peak grip force and average grip force during transport were higher for the less-affected hand $(F[1,6]=6.75$, $P<0.05$, and $F[1,6]=12.40, P<0.05$, respectively; $6 / 7$ participants, exception participant $\mathrm{KM}$ ) compared to the affected hand. Likewise, peak and mean grip force were higher for the heavy compared to the light weight $(F[1,6]=36.92, P<0.001$, and $F[1,6]=70.66, P<0.001$; all participants). Post hoc analysis of the hand $\times$ weight interaction on average grip force during transport $(F[1,6]=7.05, \quad P<0.05$; all participants $)$ revealed that

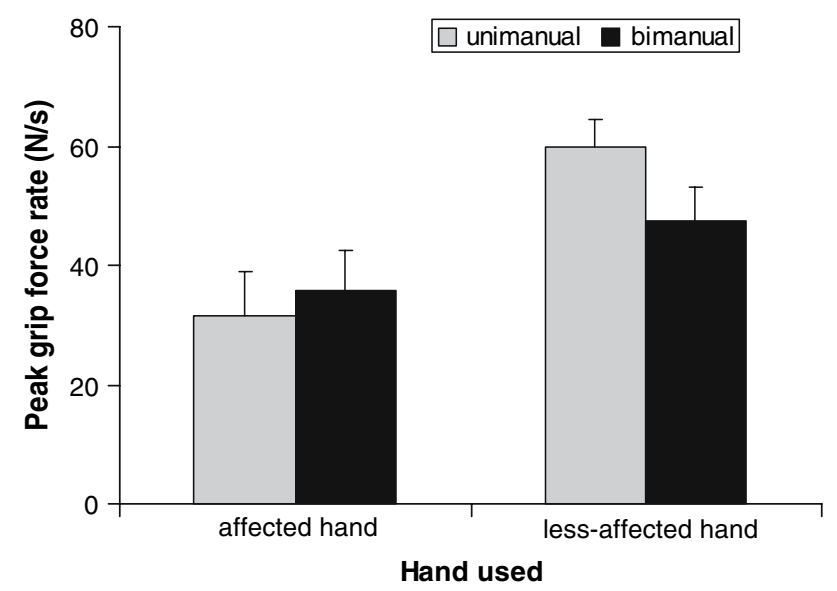

Fig. 6 Graphical display of the hand $\times$ condition interaction that was found for peak grip force rate. Error bars represent standard error weight of the object did not significantly affect mean grip force of the affected hand $(5.03$ and $6.66 \mathrm{~N}$ for the 250 and $500 \mathrm{~g}$ objects, respectively), but it did affect average grip force of the less-affected hand $(7.20$ and $11.22 \mathrm{~N}$ for the 250 and $500 \mathrm{~g}$ objects, respectively; 6/7 participants, exception participant $\mathrm{KM})$. No effects were present on the variability of grip force.

When the bimanual-equal and bimanual unequal weight conditions were compared, a main effect of weight on both peak grip force $(F[1,6]=6.70, P<0.05$; all participants $)$ and average grip force during transport $(F[1,6]=8.19, P<0.05$; all participants) were found. In both cases, values were higher for heavier weights. Finally, a condition $\times$ hand interaction $(F[1,6]=8.80, P<0.05)$ was found for the coefficient of variation. For the affected hand, the coefficient of variation was higher than that of the less-affected hand and it increased from equal to unequal weight conditions (25.20-26.32\%), while it decreased from equal to unequal weight conditions in the less-affected hand (20.90-18.93\%; 5/7 participants, exception participants KD and $\mathrm{KM})$.

\section{Discussion}

In the present study, we examined unimanual and bimanual fingertip force control in children with hemiplegic CP. Specifically, we studied the effects of lifting objects with one hand or with both hands together in order to examine the effect on temporal characteristics and on fingertip force control for each hand. This was done to determine whether any benefit exists for the affected hand when it performs the task concurrently with the less-affected hand. Our main finding was that such bimanual facilitation exists for some of the force variables, but that the temporal aspects of the task were generally adversely affected by bimanual control. These results and their implications are discussed in detail below.

Bimanual task performance: facilitation of the more-affected side?

Our main question in the present study, "does bimanual task performance facilitate the performance of the affected hand?" was inspired by four separate lines of research. First, some experimental studies have shown such a benefit of bimanual performance during grasping (e.g., Sugden and Utley 1995), while others failed to do so (e.g., Steenbergen et al. 2000) during reaching. Second, neurological evidence suggests predominantly contralateral control of the distal fingers (Colebatch and Gandevia 1989), although ipsilateral activation in M1 may be present in some contexts (e.g., Donchin et al. 2002). Third, it has been shown that 
performance related to fingertip force control can be improved in the affected hand if movements are first performed with the less-affected hand (Gordon et al. 1999, 2006a). Finally, bimanual training is increasingly being used as a treatment approach (Charles and Gordon 2006), and therefore a better understanding of how the two hands interact is required to best direct such rehabilitation approaches.

In the present study, children with hemiplegic CP lifted an object with each hand either unimanually, or bimanually. In the latter conditions, the weight of the two objects was either equal or unequal. Two analyses were performed that informed us, (1) whether a benefit was gained by the affected hand when it lifted an object while the less-affected hand concurrently lifted an object of the same weight, (2) whether interference effects between both hands were present when lifting two objects with unequal weight bimanually.

With respect to the temporal sequencing of forces, as assessed by the durations of seven phases (see Fig. 3a-c), we found that there is a (temporal) cost of lifting bimanually. First, this was evidenced by increased durations of each of these phases in the bimanual conditions as compared to the unimanual conditions. Second, we found unidirectional adaptation when unimanual and bimanual conditions were compared (see Fig. 3a compared to Fig. 3b,c). Here, the less-affected hand slowed down its duration to a large degree to match that of the affected hand under bimanual conditions. Concurrently with this, there was also a slight slowing down of the affected hand during bimanual conditions. Thus, moving bimanually had a negative effect on the total duration of the task for both hands. These findings are in line with some existing studies on unimanual and bimanual movement control in children with hemiplegic CP. Specifically, the unidirectional adaptation that we found, that is, the slowing down of the less-affected hand in the bimanual conditions to match that of the affected hand, was shown before in tasks that demanded participants to move as fast as possible (e.g., Steenbergen et al. 1996, 2000; Utley and Sugden 1998). However, we did instruct participants to move at their own selected speed. Previous studies using the same instruction have shown some benefit, i.e., speeding up of the affected hand during bimanual control (e.g., Sugden and Utley 1995). This begs the question as to what may explain the lack of bimanual benefit for the temporal aspects of the task in the present study. The most likely explanation is that the present task predominantly involved distal musculature in contrast to previous studies that used reaching movements in which predominantly the proximal musculature was involved. Evidently, the present results suggest that, at least temporally, no facilitation is present at the affected hand when moving bimanually. This finding may illustrate the behavioural consequence of the neurophysiological findings that indicate a marginal input from bilateral pathways to the distal musculature (e.g., Colebatch and Gandevia 1989).

With respect to the force-related variables we found effects of bimanual movement conditions on the two force related variables, grip force at load force onset and peak grip force rate. For both variables, the differences between the two hands that were present in the unimanual conditions were reduced during bimanual conditions. Grip force at load force onset was similar for the affected $(3.1 \mathrm{~N})$ and less-affected hand $(3.3 \mathrm{~N})$ during bimanual conditions (see Fig. 5). This was also found for peak grip force rates in the bimanual conditions, but to a less degree (affected, $35.9 \mathrm{~N} / \mathrm{s}$; less-affected, $47.6 \mathrm{~N} / \mathrm{s}$, see Fig. 6) compared to what was found for grip force at load force onset. Although most of the reduction in between-hand difference was attributable to adaptation of the less-affected hand, the affected hand also contributed to this reduction. Thus, it is not the case that one hand 'enslaves' the other, that is to say, there is unidirectional adaptation, as we found for the temporal aspects of the task described above and what was previously found (e.g., Steenbergen et al. 1996, 2000). Rather, the present results suggest a form of asymmetrical mutual adaptation, primarily, but not exclusively, established by the lessaffected hand. Importantly, these findings indicate that bimanual movements may help the affected hand to produce more 'regular', or fine, force control.

Still, no facilitation of force control was found for the three force-related variables during transport. Peak grip force and average grip force during transport were consistently higher when the heavier objects were lifted. In addition, for average grip force during transport, this effect was primarily present at the less-affected hand. No differential weight effects were found for the affected side. Surprisingly, despite equal or higher grip forces in the affected hand at load force onset, the peak and average grip force were lower on the affected side for both the unimanual and bimanual-equal weight conditions. This finding contradicts previous studies of grip and lift showing increased values when participants with hemiplegic unimanually statically hold an object with the affected hand (cf. Eliasson et al. 1991, Gordon and Duff 1999). There may be two potential reasons for this is difference in findings, one related to the task and a methodological one. First, in our task we asked subjects to dynamically transport the object instead of statically hold it. This difference in task contexts may have affected the findings on grip force. Second, the dynamic component of our task was also a potential caveat as higher grip forces are required during faster accelerations. Therefore, the decreased speed with which the object is lifted in the unimanual conditions with the affected hand may have resulted in lower inertial forces acting on the object. However, peak grip force and average grip force during 
transport were also lower in the bimanual equal-weight conditions where there was synchronization of both hands. Thus, it is unlikely that the lower grip force measures in the affected hand were due to the speed of lifting. Rather, it appears that task differences between this study and previous one may have contributed to the different findings on grip force control during transport at the affected side. This possibility requires further study.

In sum, anticipation and sequencing of force control (as evidenced by peak grip force rate and grip force at load force onset, respectively) were facilitated at the affected side when it moved together with the less-affected side. These improvements in force control did not lead to quicker movement performance of the affected side during bimanual movements. Grip force control of the affected side during bimanual transport was also unaffected.

\section{Mechanisms underlying bimanual coupling}

As our study was primarily aimed at examining the effects of bimanual performance on fingertip force control of the affected hand rather than uncovering the central or peripheral control mechanisms, we did not perform neuroimaging, and consequently have no detailed lesion data. Therefore, we can only speculate on the possible neural control mechanisms that may explain our findings.

The maturation of the corpus callosum, the major fiber bundle connecting the two hemispheres, has been linked with age-related changes in temporal and spatial bimanual coordination (Jeeves et al. 1988; Njiokiktjien et al. 1986). In this respect, Fagard et al. (2001) showed that the development of interhemispheric communication was congruent with a behaviourally observed improvement in bimanual coordination. Furthermore, research in primates showed ipsilateral motor cortex activation during hand movements (e.g., Aizawa et al. 1990; Donchin et al. 2002). The functional role of this ipsilateral activity, however, remains unclear (see Carson 2005 for a review of neural mechanisms potentially mediating bilateral interactions). Still, a marked development or persistence of ipsilateral motor pathways has been shown in part of the population with congenital hemiparesis (e.g., Staudt et al. 2004; Nezu et al. 1999). It has been suggested that the utilization of these pathways may be detrimental to functional recovery in stroke patients (Werhahn et al. 2003). Explanation of the functionality is further complicated by the finding that $10-30 \%$ of fibers in the lateral corticospinal tract remain uncrossed at the motor decussation (Nathan and Smith 1973) and that there is an occurrence of bilateral lesions in approximately one-third of individuals with hemiparetic CP (Okumura et al. 1997). Thus, to date, there is remarkably little consensus concerning the neural basis of bilateral facilitation of the more-affected extremity.
Clinical practice

For two of the five force-related variables where bimanual coupling was found, some advantage was gained for the affected hand. This was present for grip force at load force onset, and for peak grip force rate. This finding suggests that for these force aspects the affected hand may indeed benefit from actions that are performed concurrently with the other hand (viz., under bimanual conditions), especially when these actions are intensively practiced. However, grip force control of the affected hand during object transport was not affected by moving together with the less-affected hand. One of the obvious consequences of congenital hemiplegia is that individuals are tempted to 'learn' to only use the less-affected side for the performance of activities of daily living, the so-called 'learned non-use' (Taub et al. 1999). A currently influential therapeutic intervention aimed at overcoming this learned disuse is ConstraintInduced Movement Therapy (CIMT) (e.g., Taub and Wolf 1997; Wolf et al. 2006). In its most basic form, CIMT restrains movements of the less-affected side, while at the same time providing intensive practice of the affected side through shaping and repetitive practice. Several studies have shown that intensive practice associated with CIMT can improve movement efficiency and functional limitations among a carefully selected subgroup of children with hemiplegic CP of varying ages (e.g., Taub et al. 2004 DeLuca et al. 2003, Eliasson et al. 2005, Charles and Gordon 2005, Gordon et al. 2006b). However, as Gordon et al. (2006b) noted, it is not very likely that children with hemiplegia would continue to perform the unimanual activities with their affected hand once the restraint is removed. Thus, an obvious question is whether such gains would be better achieved by practicing with both hands. Indeed, recent developments in rehabilitation show that bimanual, or bilateral, training may facilitate performance and rehabilitation of the affected hand in a more 'natural' manner (Charles and Gordon 2006; Gordon et al. 2007). Similar results have been obtained in stroke patients (e.g., Cauraugh 2004; Cauraugh and Summers 2005; Luft et al. 2004; Mudie and Matyas 2000; Whitall et al. 2000; for a review of the positive effect of bilateral movement training for post-stroke motor rehabilitation, see Stewart et al. 2006). Based on our findings of a marginal benefit of force control for the affected side during bimanual conditions, a major challenge, in light of the apparent efficacy of bimanual therapy, is to determine the extent to which 'distal control' can be (re)gained by the affected hand when bimanual tasks are intensively trained. In addition, it may also be established how task context (e.g., holding versus moving) may differentially affect or facilitate grip force control of the affected hand. As the present study had few participants and did not provide enough practice to determine whether actual 
training under bimanual conditions would be beneficial, further study into this aspect of rehabilitation is warranted.

Limitations and future directions

The number of participants in the present study $(n=7)$ was relatively small. Despite the small number of participants, the present study showed significant results in which the direction of the effects was present in six, and sometimes all of the participants. In most instances it was the youngest participant that showed an opposite effect. Thus, the results appear to be robust, but at the same time suggest a critical age for them to show up. Therefore, future studies with a larger sample size and narrower sample selection criteria, such as age range, may be required to further elucidate these findings. These studies will be important as they may be used to guide rehabilitation strategies.

Acknowledgements The authors like to express their gratitude to the children and parents who kindly participated in this study. The study was supported by a grant from the United Cerebral Palsy Research and Education Foundation. Finally, we thank Prof. Richard Carson for useful suggestions on an earlier draft of this manuscript.

Open Access This article is distributed under the terms of the Creative Commons Attribution Noncommercial License which permits any noncommercial use, distribution, and reproduction in any medium, provided the original author(s) and source are credited.

\section{References}

Aizawa H, Mushiake H, Inase M, Tanji J (1990) An output zone of the monkey primary motor cortex specialized for bilateral hand movement. Exp Brain Res 82:219-221

Brinkman J, Kuypers HGJ (1973) Cerebral control of contralateral and ipsilateral arm, hand and finger movements in split-brain rhesusmonkey. Brain 96:653-674

Brown JV, Schumacher U, Rohlman A, Ettlinger G, Schmidt RC, Skreczek W (1989) Aimed movements to visual targets in hemiplegic and normal children: is the "good" hand of children with infantile hemiplegia also normal? Neuropsychologia 27:283-302

Carson RG (2005) Neural pathways mediating bilateral interactions between the upper limbs. Brain Res Rev 49(3):641-662

Cauraugh JH (2004) Coupled rehabilitation protocols and neural plasticity: upper extremity improvements in chronic hemiparesis. Restor Neurol Neurosci 22:337-347

Cauraugh JH, Summers JJ (2005) Neural plasticity and bilateral movements: a rehabilitation approach for chronic stroke. Progr Neurobiol 75:309-320

Chang J, Wu T, Wu W, Su F (2005) Kinematical measure for spastic reaching in children with cerebral palsy. Clinic Biomech 20:381388

Charles J, Gordon AM (2005) A critical review of constraint-induced movement therapy and forced-use in children with hemiplegia. Neural Plast 12:245-262

Charles J, Gordon AM (2006) Development of hand-arm bimanual intensive therapy (HABIT) for improving bimanual coordination in children with hemiplegic cerebral palsy. Dev Med Child Neurol 48:931-936
Colebatch JG, Gandevia SC (1989) The distribution of muscular weakness in upper motor neuron lesions affecting the arm. Brain 112:749-763

DeLuca SC, Echols K, Ramey SL, Taub E (2003) Pediatric constraintinduced movement therapy for a young child with cerebral palsy: two episodes of care. Phys Ther 83:1003-1013

Donchin O, Gribova A, Steinberg O, Mitz AR, Bergman H, Vaadia E (2002) Single-unit activity related to bimanual arm movements in the primary and supplementary motor cortices. J Neurophys 88:3498-3517

Duff SV, Gordon AM (2003) Learning of grasp control in children with hemiplegic cerebral palsy. Dev Med Child Neurol 45:746-757

Eliasson AC, Gordon AM, Forssberg H (1991) Basic co-ordination of manipulative forces of children with cerebral palsy. Dev Med Child Neurol 33:661-670

Eliasson AC, Gordon AM, Forssberg H (1992) Impaired anticipatory control of isometric forces during grasping by children with cerebral palsy. Dev Med Child Neurol 34(3):216-225

Eliasson AC, Krumlinde-Sundholm L, Shaw K, Wang C (2005) Effects of constraint-induced movement therapy in young children with hemiplegic cerebral palsy: an adapted model. Dev Med Child Neurol 47:266-275

Fagard J, Hardy-Léger I, Kervella C, Marks A (2001) Changes in interhemispheric transfer rate and the development of bimanual coordination during childhood. J Exp Child Psychol 80:1-22

Forssberg H, Eliasson AC, Redon-Zouitenn C, Mercuri E, Dubowitz L (1999) Impaired grip-lift synergy in children with unilateral brain lesions. Brain 122:1157-1168

Gordon AM, Schneider JA, Chinnan A, Charles J (2007) Efficacy of Hand-arm bimanual intensive therapy (HABIT) in children with cerebral palsy. Dev Med Child Neurol 49:830-838

Gordon AM, Steenbergen B (in press) Bimanual coordination in children with cerebral palsy. In: Eliasson AC, Burtner P (eds) Child with cerebral palsy: management of the upper extremity. Clinics in Developmental Medicine. MacKeith Press, London

Gordon AM, Charles J, Duff SV (1999) Fingertip forces during object manipulation in children with hemiplegic cerebral palsy. II: bilateral coordination. Dev Med Child Neurol 41:176-185

Gordon AM, Duff SV (1999) Relation between clinical measures and fine manipulative control in children with hemiplegic cerebral palsy. Dev Med Child Neurol 41:586-591

Gordon AM, Charles J, Steenbergen B (2006a) Fingertip force planning during grasp is disrupted by impaired sensorimotor integration in children with hemiplegic cerebral palsy. Ped Res 60:587-591

Gordon AM, Charles J, Wolf SL (2006b) Efficacy of contraint-induced movement therapy on more-affected upper-extremity use in children with hemiplegic cerebral palsy is not age-dependent. Pediatrics 117:363-373

Jebsen RH, Taylor N, Trieschmann RB (1969) Objective and standardized test of hand function. Arch Phys Med Rehab (Chicago) 50:311

Jeeves MA, Silver PH, Milne AB (1988) Role of the corpus callosum in the development of a bimanual motor skill. Dev Neuropsych 4(4):305-323

Kaufman AS, Kaufman NL (1990) Kaufman brief intelligence test. American Guidance Services, Circle Pines, MN

Kelso JAS, Southard DL, Goodman D (1979) On the nature of human interlimb coordination. Science 203:1029-1031

Krumlinde-Sundholm L, Eliasson AC (2003) Development of the assisting hand assessment: a Rasch-built measure intended for children with unilateral upper limb impairments. Dev Med Child Neurol 10:16

Krumlinde-Sundholm L, Holmefur M, Kottorp A, Eliasson AC (2007) The assisting hand assessment: current evidence of validity, reliability, and responsiveness to change. Dev Med Child Neurol 49:259-264 
Kuhtz-Buschbeck JP, Sundholm LK, Eliasson AC, Forssberg H (2000) Quantitative assessment of mirror movements in children and adolescents with hemiplegic cerebral palsy. Dev Med Child Neurol 42(11):728-736

Luft AR, McCombe-Waller S, Whitall J, Forrester LW, Macko R, Sorkin JD, Schulz JB, Goldberg AP, Hanley DF (2004) Repetitive bilateral arm training and motor cortex activation in chronic stroke-a randomized controlled trial. J Am Med Assoc 292(15):1853-1861

Mackinnon SE, Dellon AL (1985) Two-point discrimination tester. J Hand Surg [Am] 10:906-907

Mudie MH, Matyas TA (2000) Can simultaneous bilateral movement involve the undamaged hemisphere in reconstruction of neural networks damaged by stroke? Dis Reh 22(1-2):23-37

Mutsaarts M, Steenbergen B, Bekkering H (2006) Anticipatory planning deficits and task context effects in hemiparetic cerebral palsy. Exp Brain Res 172(2):151-162

Nathan PW, Smith MC (1973) Effects of 2 unilateral cordotomies on motility of lower limbs. Brain 96:471-494

Nezu A, Kimura S, Takeshita S, Tanaka M (1999) Functional recovery in hemiplegic cerebral palsy: ipsilateral electromyographic responses to focal transcranial magnetic stimulation. Brain Dev 21(3):162-165

Njiokiktjien C, Driessen M, Habraken L (1986) Development of supination-pronation movements in normal children. Hum Neurobiol 5:199-203

Okumura A, Kato T, Kuno K, Hayakawa F, Watanabe K (1997) MRI findings in patients with spastic cerebral palsy. 2. Correlation with type of cerebral palsy. Dev Med Child Neurol 39:369-372

Roon van D, Steenbergen B, Meulenbroek RGJ (2004) Trunk recruitment during spoon use in tetraparetic cerebral palsy. Exp Brain Res 155:186-195

Sugden D, Utley A (1995) Interlimb coupling in children with hemiplegic cerebral palsy. Dev Med Child Neurol 37:293-309

Staudt M, Gerloff C, Grodd W, Holthausen H, Niemann G, KragelohMann I (2004) Reorganization in congenital hemiparesis acquired at different gestational ages. Ann Neurol 56:854-863

Steenbergen B, Hulstijn W, de Vries A, Berger M (1996) Bimanual movement coordination in spastic hemiparesis. Exp Brain Res 110:91-98

Steenbergen B, Van Thiel E, Hulstijn W, Meulenbroek RGJ (2000) The coordination of reaching and grasping in spastic hemiparesis. Hum Mov Sci 19:75-105
Steenbergen B, Gordon AM (2006) Activity limitation in hemiplegic cerebral palsy: evidence for disorders in motor planning. Dev Med Child Neurol 48:780-783

Stewart KC, Cauraugh JH, Summers JJ (2006) Bilateral movement training and stroke rehabilitation: a systematic review and metaanalysis. J Neurol Sci 244(1-2):89-95

Taub E, Wolf SL (1997) Constraint induction techniques to facilitate upper extremity use in stroke patients. Top Stroke Rehab 3:38-61

Taub E, Uswatte G, Pidikiti R (1999) Constraint-induced movement therapy: a new family of techniques with broad application to physical reahbilitation-a clinical review. J Rehab Res Dev 36:237-251

Taub E, Ramey SL, DeLuca S, Echols K (2004) Efficacy of constraintinduced movement therapy for children with cerebral palsy with asymmetric motor impairment. Pediatrics 113:305-312

Thiel van E, Steenbergen B (2001) Shoulder and hand displacements during hitting, reaching, and grasping movements in hemiparetic cerebral palsy. Mot Control 2:72-88

Thiel van E, Meulenbroek RGJ, Smeets JBJ, Hulstijn W (2002) Fast adjustments of ongoing movements in hemiparetic cerebral palsy. Neuropsychologia 40:16-27

Utley A, Sugden DA (1998) Interlimb coupling in children with hemiplegic cerebral palsy during reaching and grasping at speed. Dev Med Child Neurol 40:396-404

Utley A, Steenbergen B (2006) Discrete bimanual co-ordination in children and young adolescents with hemiparetic cerebral palsy: recent findings, implications and future research directions. Ped Rehab 9(2):127-136

Volman MJM, Wijnroks A, Vermeer A (2002) Bimanual circle drawing in children with spastic hemiparesis: effect of coupling modes on the performance of the impaired and unimpaired arms. Acta Psychol 110(2-3):339-356

Werhahn KJ, Conforto AB, Kadom N, Hallet M, Cohen LG (2003) Contribution of the ipsilateral motor cortex to recovery after chronic stroke. Ann Neurol 54:464-472

Whitall J, Waller SM, Silver KHC, et al (2000) Repetitive bilateral arm training with rhythmic auditory cueing improves motor function in chronic hemiparetic stroke. Stroke 31(10):2390-2395

Wolf SL, Winstein CJ, Miller JP, Taub E, Uswatte G, Morris D, Giuliani C, Light KE, Nichols-Larsen D (2006) Effect of constraint-induced movement therapy on upper extremity function 3 to 9 months after stroke: the EXCITE randomized clinical trial. J Am Med Assoc 296:2095-2104 